\title{
Complete genome sequence of Slackia heliotrinireducens type strain (RHS $1^{\mathrm{T}}$ )
}

\author{
Rüdiger Pukall ${ }^{1}$, Alla Lapidus ${ }^{2}$, Matt Nolan², Alex Copeland ${ }^{2}$, Tijana Glavina Del Rio ${ }^{2}$, Susan \\ Lucas $^{2}$, Feng Chen ${ }^{2}$, Hope Tice ${ }^{2}$, Jan-Fang Cheng ${ }^{2}$, Olga Chertkov ${ }^{2,3}$, David Bruce ${ }^{2,3}$, Lynne \\ Goodwin $^{2,3}$, Cheryl Kuske ${ }^{2,3}$, Thomas Brettin ${ }^{2,3}$, John C. Detter ${ }^{2,3}$, Cliff Han ${ }^{2,3}$, Sam Pitluck ${ }^{2}$, \\ Amrita Pati ${ }^{2}$, Konstantinos Mavrommatis ${ }^{2}$, Natalia Ivanova ${ }^{2}$, Galina Ovchinnikova ${ }^{2}$, Amy \\ Chen $^{4}$, Krishna Palaniappan ${ }^{4}$, Susanne Schneider ${ }^{1}$, Manfred Rohde ${ }^{5}$, Patrick Chain ${ }^{2,6}$, Pa- \\ trikD'haeseleer ${ }^{2,6}$, Markus Göker1 ${ }^{1}$, James Bristow ${ }^{2}$, Jonathan A. Eisen ${ }^{2,7}$, Victor Markowitz ${ }^{4}$, \\ Nikos C. Kyrpides ${ }^{2}$, Hans-Peter Klenk ${ }^{1}$, and Philip Hugenholtz ${ }^{2 *}$ \\ ${ }^{1}$ DSMZ - German Collection of Microorganisms and Cell Cultures GmbH, Braunschweig, \\ Germany \\ 2 DOE Joint Genome Institute, Walnut Creek, California, USA \\ ${ }^{3}$ Los Alamos National Laboratory, Bioscience Division, Los Alamos, New Mexico, USA \\ ${ }^{4}$ Biological Data Management and Technology Center, Lawrence Berkeley National Labora- \\ tory, Berkeley, California, USA \\ ${ }^{5}$ HZI - Helmholtz Centre for Infection Research, Braunschweig, Germany \\ ${ }^{6}$ Lawrence Livermore National Laboratory, Livermore, California, USA \\ ${ }^{7}$ University of California Davis Genome Center, Davis, California, USA
}

*Corresponding author: Philip Hugenholtz

Keywords: Gram-positive coccus, anaerobic, asaccharolytic, pyrrolizidine alkaloids, Coriobacteriaceae

Slackia heliotrinireducens (Lanigan 1983) Wade et al. 1999 is of phylogenetic interest because of its location in a genomically yet uncharted section of the family Coriobacteriaceae, within the deep branching Actinobacteria. Strain RHS $1^{\top}$ was originally isolated from the ruminal flora of a sheep. It is a proteolytic anaerobic coccus, able to reductively cleave pyrrolizidine alkaloids. Here we describe the features of this organism, together with the complete genome sequence, and annotation. This is the first complete genome sequence of the genus Slackia, and the 3,165,038 bp long single replicon genome with its 2798 protein-coding and 60 RNA genes is part of the Genomic Encyclopedia of Bacteria and Archaea project.

\section{Introduction}

Strain RHS $1^{\mathrm{T}}$ (= DSM $20476=$ ATCC $29202=\mathrm{JCM}$ 14554 ) is the type strain of the species Slackia heliotrinireducens and was originally described by Lanigan in 1976 as Peptococcus heliotrinreducans (sic) [1] and validly published following an orthographic correction as Peptococcus heliotrinreducens in 1983 [2,3]. The strain was later transferred to the genus Peptostreptococcus on the basis of its $\mathrm{G}+\mathrm{C}$ content [4]. 16S rRNA gene sequence analysis indicated that it should not be assigned to the genus Peptostreptococcus and therefore the strain was subsequently allocated to the novel genus Slackia as $S$. heliotrinireducens $[5,6]$. The three species of the genus Slackia, S. exigua, S. faecicanis, and $S$. heliotrinireducens form a distinct cluster within the Coriobacteriaceae, located in the neigh- borhood to the genera Cryptobacterium and Collinsella.

Five additional strains identified as $S$. heliotrinireducens based on their proteolytic enzyme profiles have been isolated from human polymicrobial abscesses [7], but these strains were dissimilar from the type strain as shown by pyrolysis mass spectrometry [8]. With 94\% sequence identity (16S rRNA gene), S. exigua, the type strain of the closest related species represents the only meaningful (>91\%) hit in nucleotide sequence database searches, indicating a complete lack of cultivated and even uncultivated relatives of strain RHS $1^{\mathrm{T}}$ in accessible microbiological diversity. Screening of environmental samples and surveys reported at NCBI BLAST server indicated no closely related 
phylotypes that can be linked to the species (as of July 2009). Here we present a summary classification and a set of features for $S$. heliotrinireducens RHS $1^{\mathrm{T}}$ Together with the description of the complete genomic sequencing and annotation.

\section{Classification and features}

Figure 1 shows the phylogenetic neighborhood of $S$. heliotrinireducens strain RHS $1^{\mathrm{T}}$ in a 16S rRNA based tree. The sequence of one of the two $16 \mathrm{~S}$ rRNA genes differs in two nucleotides from the other copy and from the previously published $16 \mathrm{~S}$ rRNA sequence generated from ATCC 29202 (AF101241).

S. heliotrinireducens is Gram-positive, nonmotile, obligately anaerobic, and does not produce endospores (Table 1). Strain RHS 1 forms cocci or coccobacilli (Figure 2) with a diameter of 0.3 to 0.6 $\mu \mathrm{m}$ and $0.8 \times 1.2 \mu \mathrm{m}$, respectively [5,6]. The strain grows very slowly on blood agar and forms small translucent, glistening colonies, up to $1 \mathrm{~mm}$ in diameter after extensive incubation. It does not util- ize carbohydrates, but reduces nitrates and pyrrolizidine alkaloids $[5,6]$. Reductive cleavage of pyrrolizidines (heliotrine, europine, heleurine, supinine and lasiocarpine) occurs by using hydrogen gas or formate as hydrogen donor [1]. Ammonia is formed from tryptone, yeast extract, adenine, uracil and arginine. Nitrates are completely reduced to ammonia if an appropriate electron donor $\left(\mathrm{H}_{2}\right.$, formate) is present [19]. The strain is bilesensitive, indole-negative, hydrolyses arginine but not esculin. Does not produce catalase or urease, but is able to dissimilate arginine. Growth is generally stimulated by addition of $0.5 \%$ arginine. Metabolic products from $S$. heliotrinireducens grown in pre-reduced PYG broth are acetic acid, isovaleric acid, and butyric acid in trace amounts [4].

Almost nothing is known about the chemotaxonomy of strain RHS $1^{\mathrm{T}}$, except that its predominant cellular fatty acid is C18:1 [4].

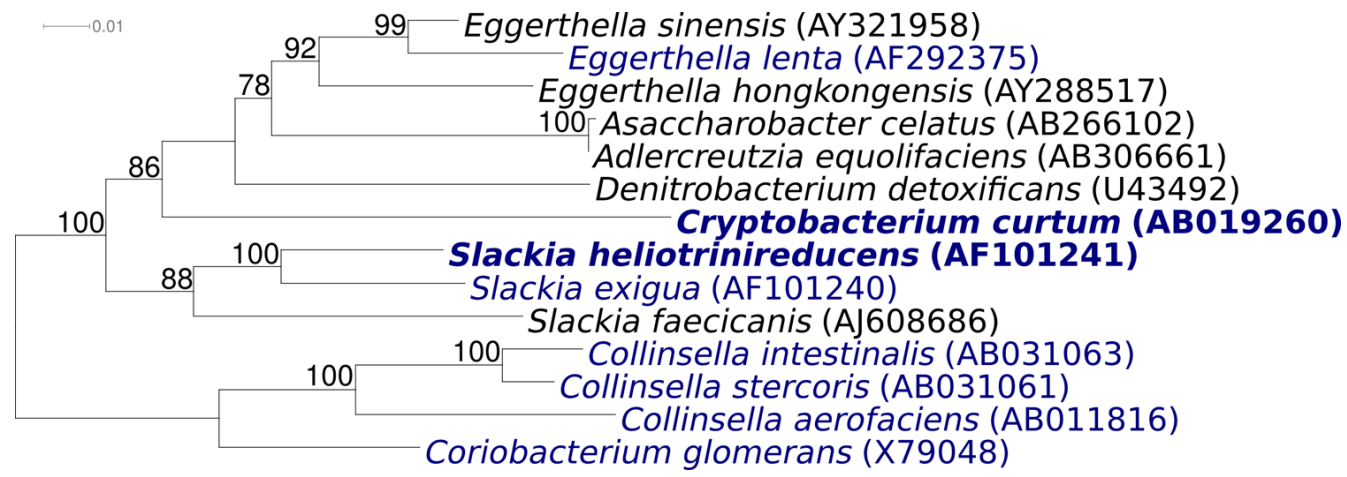

Figure 1. Phylogenetic tree highlighting the position of $S$. heliotrinireducens $\mathrm{RHS} 1^{\top}$ relative to other type strains within the family Coriobacteriaceae. The tree was inferred from 1,422 aligned $16 \mathrm{~S}$ rRNA characters $[9,10]$ under the maximum likelihood criterion [11], and rooted with type strains of the genera Collinsella and Coriobacterium. The branches are scaled in terms of the expected number of substitutions per site. Numbers above branches are support values from 1,000 bootstrap replicates, if larger than $60 \%$. Lineages with type strain genome sequencing projects registered in GOLD [12] are shown in blue, published genomes in bold, e.g. the recently published GEBA genomes from Cryptobacterium curtum [13], and Eggerthella lenta [14].

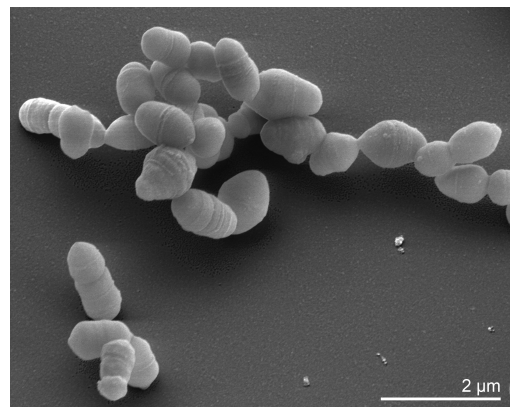

Figure 2. Scanning electron micrograph of S. heliotrinireducens RHS $1^{\top}$ 
Table 1. Classification and general features of $S$. heliotrinireducens RHS $1^{\top}$ according to the MIGS recommendations [15].

\begin{tabular}{|c|c|c|c|}
\hline MIGS ID & Property & Term & Evidence code \\
\hline & \multirow{9}{*}{ Current classification } & Domain Bacteria & $\overline{T A S}[16]$ \\
\hline & & Phylum Actinobacteria & TAS [17] \\
\hline & & Class Actinobacteria & TAS [18] \\
\hline & & Order Coriobacteriales & TAS [18] \\
\hline & & Suborder Coriobacteridae & TAS [18] \\
\hline & & Family Coriobacteriaceae & TAS [18] \\
\hline & & Genus Slackia & TAS [5] \\
\hline & & Species Slackia heliotrinireducens & TAS [5] \\
\hline & & Type strain RHS 1 & TAS [5] \\
\hline & Gram stain & positive & TAS [1] \\
\hline & Cell shape & cocci to coccobacilli & TAS [1] \\
\hline & Motility & nonmotile & TAS [1] \\
\hline & Sporulation & nonsporulating & TAS [1] \\
\hline & Temperature range & mesophile, $30-46^{\circ} \mathrm{C}$ & TAS [19] \\
\hline & Optimum temperature & $38-42^{\circ} \mathrm{C}$ & TAS [19] \\
\hline & Salinity & $5 \mathrm{~g} \mathrm{NaCl}$ per I & TAS [5] \\
\hline \multirow[t]{3}{*}{ MIGS-22 } & Oxygen requirement & obligate anaerobic & TAS [1] \\
\hline & Carbon source & asaccharolytic & TAS [1] \\
\hline & Energy source & arginine, proteolytic & NAS \\
\hline MIGS-6 & Habitat & rumen (sheep) & TAS [1] \\
\hline MIGS-15 & Biotic relationship & free living & NAS \\
\hline \multirow[t]{3}{*}{ MIGS-14 } & Pathogenicity & assumed & NAS \\
\hline & Biosafety level & $1(+)$ & TAS [20] \\
\hline & Isolation & rumen of sheep & TAS [1] \\
\hline MIGS-4 & Geographic location & Australia & NAS \\
\hline MIGS-5 & Sample collection time & about 1974 & TAS [1] \\
\hline $\begin{array}{l}\text { MIGS-4.1 } \\
\text { MIGS-4.2 }\end{array}$ & Latitude - Longitude & not reported & \\
\hline MIGS-4.3 & Depth & not reported & \\
\hline MIGS-4.4 & Altitude & not reported & \\
\hline
\end{tabular}

Evidence codes - IDA: Inferred from Direct Assay (first time in publication); TAS: Traceable Author Statement (i.e., a direct report exists in the literature); NAS: Non-traceable Author Statement (i.e., not directly observed for the living, isolated sample, but based on a generally accepted property for the species, or anecdotal evidence). These evidence codes are from the Gene Ontology project [21]. If the evidence code is IDA, then the property should have been directly observed for a living isolate by one of the authors, or an expert mentioned in the acknowledgements.

\section{Genome sequencing information Genome project history}

This organism was selected for sequencing on the basis of its phylogenetic position, and is part of the Genomic Encyclopedia of Bacteria and Archaea project. The genome project is deposited in the Genome OnLine Database [12] and the complete genome sequence is in GenBank. Sequencing, finishing and annotation were performed by the DOE Joint Genome Institute (JGI). A summary of the project information is shown in Table 2.

\section{Growth conditions and DNA isolation}

S. heliotrinireducens strain RHS $1^{\mathrm{T}}$, DSM 20476, was grown anaerobically in DSMZ medium 104 (PYG) [22]; at $37^{\circ} \mathrm{C}$. DNA was isolated from 1-1.5 g of cell paste using Qiagen Genomic 500 DNA Kit (Qiagen, Hilden, Germany) with a modified protocol for cell lysis (FT), as described in Wu et al. [23]. 
Table 2. Genome sequencing project information

\begin{tabular}{|c|c|c|}
\hline MIGS ID & Property & Term \\
\hline MIGS-31 & Finishing quality & Finished \\
\hline MIGS-28 & Libraries used & $\begin{array}{l}\text { Three genomic libraries: two San- } \\
\text { ger- } 8 \mathrm{~kb} \text { pMCL200 and fosmid } \\
\text { pcc1Fos Sanger libraries and one } \\
454 \text { pyrosequence standard library }\end{array}$ \\
\hline MIGS-29 & Sequencing platforms & ABI3730, 454 GS FLX \\
\hline MIGS-31.2 & Sequencing coverage & 6x Sanger; $20 \times$ pyrosequence \\
\hline MIGS-30 & Assemblers & Newbler version 1.1.02.15, phrap \\
\hline \multirow[t]{6}{*}{ MIGS-32 } & Gene calling method & $\begin{array}{l}\text { Genemark 4.6b, GenePRIMP, } \\
\text { tRNAScan-SE-1.23, infernal } 0.81\end{array}$ \\
\hline & INSDC ID & СР001684 \\
\hline & Genbank Date of Release & August 28, 2009 \\
\hline & GOLD ID & Gc01094 \\
\hline & NCBI project ID & 20831 \\
\hline & Database: IMG-GEBA & 2500901757 \\
\hline \multirow[t]{2}{*}{ MIGS-13 } & Source material identifier & DSM 20476 \\
\hline & Project relevance & Tree of Life, GEBA \\
\hline
\end{tabular}

\section{Genome sequencing and assembly}

The genome was sequenced using a combination of Sanger and 454 sequencing platforms. All general aspects of library construction and sequencing performed at the JGI can be found on the JGI website (http://www.jgi.doe.gov/). 454 Pyrosequencing reads were assembled using the Newbler assembler version 1.1.02.15 (Roche). Large Newbler contigs were broken into 3,507 overlapping fragments of $1,000 \mathrm{bp}$ and entered into the assembly as pseudo-reads. The sequences were assigned quality scores based on Newbler consensus q-scores with modifications to account for overlap redundancy and to adjust inflated qscores. A hybrid 454/Sanger assembly was made using the parallel phrap assembler (High Performance Software, LLC). Possible mis-assemblies were corrected with Dupfinisher or transposon bombing of bridging clones [24]. Gaps between contigs were closed by editing in Consed, custom primer walk or PCR amplification. A total of 1,433 Sanger finishing reads were produced to close gaps, to resolve repetitive regions, and to raise the quality of the finished sequence. The final assembly consists of 21.045 Sanger and 205,234 pyrosequence (454) reads. Together all sequence types provided $26 \times$ coverage of the genome. The error rate of the completed genome sequence is less than 1 in 100,000.

\section{Genome annotation}

Genes were identified using GeneMark [25] as part of the genome annotation pipeline in the Inte- grated Microbial Genomes Expert Review system (http://img.jgi.doe.ogv/er) [26], followed by a round of manual curation using the JGI GenePRIMP pipeline (http://geneprimp.jgi-psf.org/) [27]. The predicted CDSs were translated and used to search the National Center for Biotechnology Information (NCBI) nonredundant database, UniProt, TIGRFam, Pfam, PRIAM, KEGG, COG, and InterPro databases. The tRNAScanSE tool [28] was used to find tRNA genes, whereas ribosomal RNAs were found by using the tool RNAmmer [29]. Other non coding RNAs were identified by searching the genome for the Rfam profiles using INFERNAL (v0.81) [30]. Additional gene prediction analysis and manual functional annotation was performed within the Integrated Microbial Genomes (http://img.jgi.doe.gov/) platform [31].

\section{Metabolic network analysis}

The metabolic Pathway/Genome Database (PGDB) was computationally generated using Pathway Tools software version 12.5 [32] and MetaCyc version 12.5 [33], based on annotated EC numbers and a customized enzyme name mapping file. It has undergone no subsequent manual curation and may contain errors, similar to a Tier 3 BioCyc PGDB [34].

\section{Genome properties}

The genome is $3,165,038$ bp long and comprises one main circular chromosome with a $60.2 \%$ GC content (Table 3 and Figure 3). Of the 2,858 genes predicted, 2,798 were protein coding genes, and 60 RNAs; 33 pseudogenes were also identified. 
The majority of the protein-coding genes (70.6\%) were assigned with a putative function, while those remaining were annotated as hypothetical proteins. The properties and the statistics of the genome are summarized in Table 3. The distribu- tion of genes into COGs functional categories is presented in Table 4, and a cellular overview diagram is presented in Figure 4, followed by a summary of metabolic network statistics shown in Table 5 .

Table 3. Genome Statistics

\begin{tabular}{lrr}
\hline Attribute & \multicolumn{1}{c}{ Value } & \% of Total \\
\hline Genome size (bp) & $3,165,038$ & $100.00 \%$ \\
DNA coding region (bp) & $2,756,714$ & $87.10 \%$ \\
DNA G+C content (bp) & $1,905,720$ & $60.21 \%$ \\
Number of replicons & 1 & \\
Extrachromosomal elements & 0 & \\
Total genes & 2,858 & $100.00 \%$ \\
RNA genes & 60 & $2.03 \%$ \\
rRNA operons & 2 & \\
Protein-coding genes & 2,798 & $97.90 \%$ \\
Pseudo genes & 33 & $1.15 \%$ \\
Genes with function prediction & 2,014 & $70.52 \%$ \\
Genes in paralog clusters & 433 & $15.15 \%$ \\
Genes assigned to COGs & 1,969 & $68.94 \%$ \\
Genes assigned Pfam domains & 1,977 & $69.22 \%$ \\
Genes with signal peptides & 562 & $19.66 \%$ \\
Genes with transmembrane helices & 123 & $4.30 \%$ \\
CRISPR repeats & 0 & \\
\hline
\end{tabular}

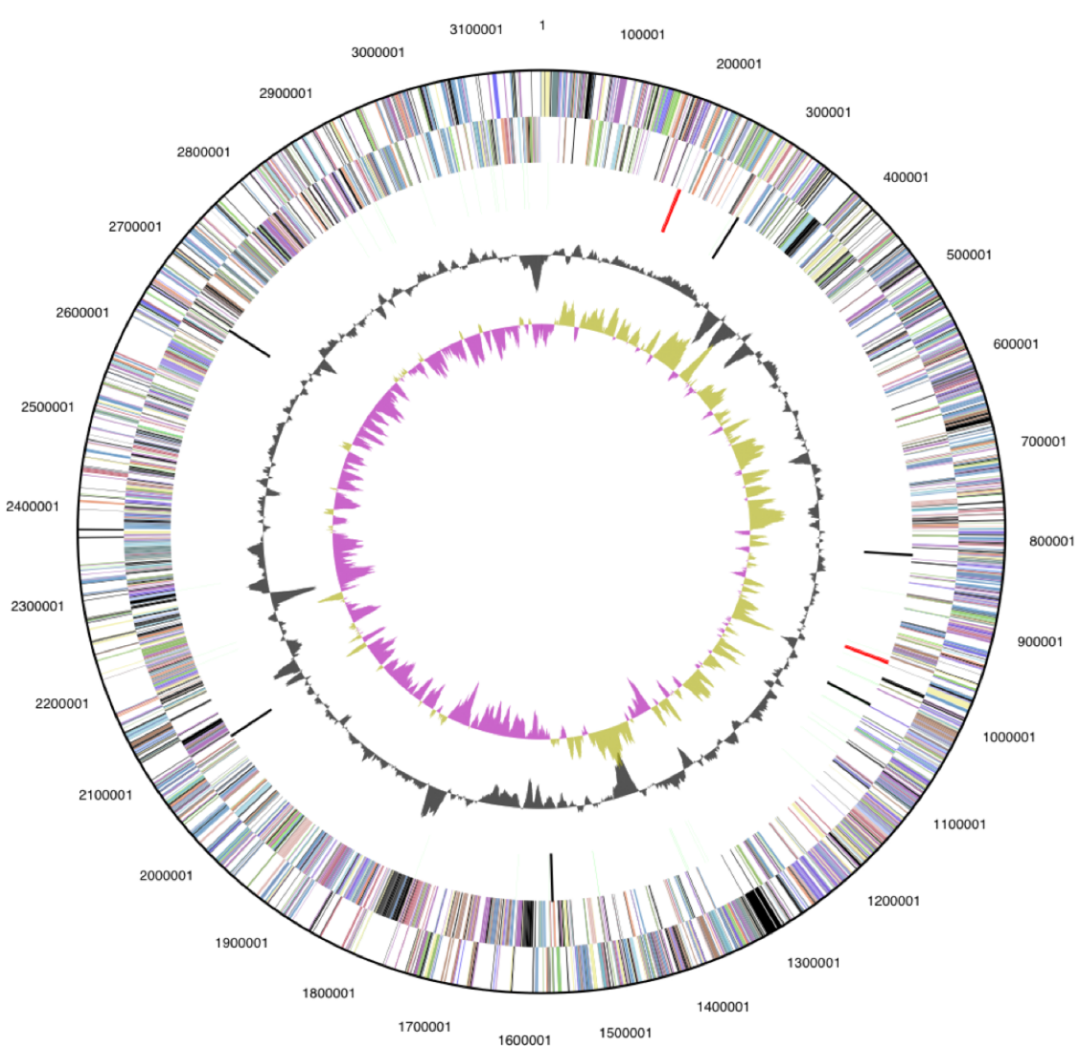

Figure 3. Graphical circular map of the genome. From outside to the center: Genes on forward strand (color by COG categories), Genes on reverse strand (color by COG categories), RNA genes (tRNAs green, rRNAs red, other RNAs black), GC content, GC skew. 
Table 4. Number of genes associated with the general COG functional categories

\begin{tabular}{lrrl}
\hline Code & Value & \% age & Description \\
\hline J & 139 & 5.0 & Translation, ribosomal structure and biogenesis \\
A & 0 & 0.0 & RNA processing and modification \\
K & 208 & 7.4 & Transcription \\
L & 134 & 4.8 & Replication, recombination and repair \\
B & 1 & 0.0 & Chromatin structure and dynamics \\
D & 25 & 0.9 & Cell cycle control, mitosis and meiosis \\
Y & 0 & 0.0 & Nuclear structure \\
V & 48 & 1.7 & Defense mechanisms \\
T & 107 & 3.8 & Signal transduction mechanisms \\
M & 93 & 3.3 & Cell wall/membrane biogenesis \\
N & 3 & 0.1 & Cell motility \\
Z & 0 & 0.0 & Cytoskeleton \\
W & 0 & 0.0 & Extracellular structures \\
U & 30 & 1.1 & Intracellular trafficking and secretion \\
O & 83 & 3.0 & Posttranslational modification, protein turnover, chaperones \\
C & 229 & 8.2 & Energy production and conversion \\
G & 68 & 2.4 & Carbohydrate transport and metabolism \\
E & 151 & 5.4 & Amino acid transport and metabolism \\
F & 58 & 2.1 & Nucleotide transport and metabolism \\
H & 109 & 3.9 & Coenzyme transport and metabolism \\
I & 66 & 2.4 & Lipid transport and metabolism \\
P & 101 & 3.6 & Inorganic ion transport and metabolism \\
Q & 33 & 1.2 & Secondary metabolites biosynthesis, transport and catabolism \\
R & 319 & 11.4 & General function prediction only \\
S & 155 & 5.5 & Function unknown \\
- & 829 & 29.6 & Not in COGs \\
\hline
\end{tabular}

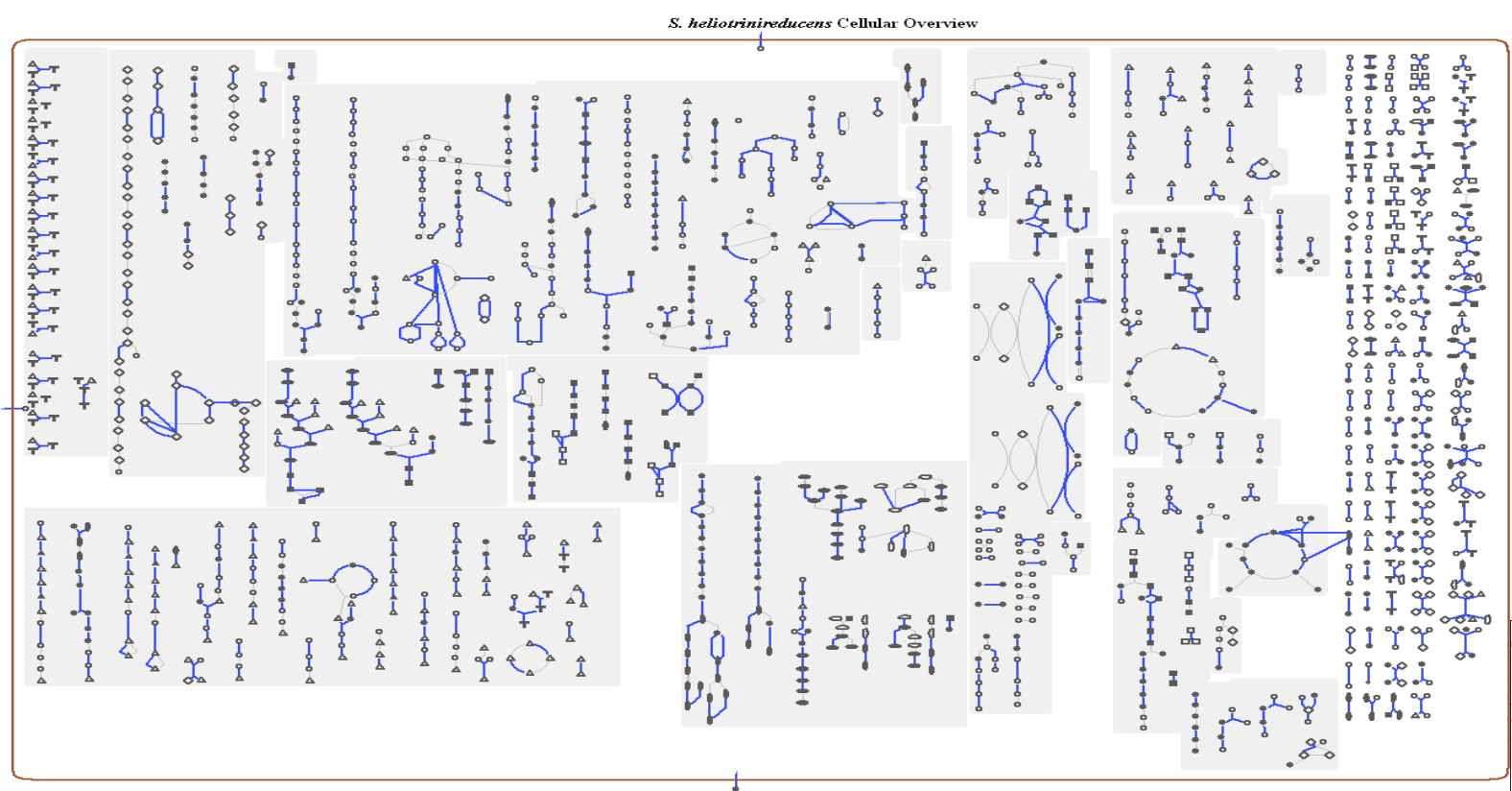

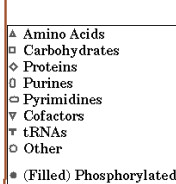

Figure 4. Schematic cellular overview diagram of all pathways of the $S$. heliotrinireducens RHS $1^{\mathrm{T}}$ metabolism. Nodes represent metabolites, with shape indicating class of metabolite (see key to right). Lines represent reactions. 
Table 5. Metabolic Network Statistics

\begin{tabular}{lr}
\hline Attribute & Value \\
\hline Total genes & 2,856 \\
Enzymes & 457 \\
Enzymatic reactions & 750 \\
Metabolic pathways & 156 \\
Metabolites & 576 \\
\hline
\end{tabular}

\section{Acknowledgements}

We would like to gratefully acknowledge the help of Gabriele Gehrich-Schröter (DSMZ) for growing S. heliotrinireducens cultures. This work was performed under the auspices of the US Department of Energy's Office of Science, Biological and Environmental Research Program, and by the University of California, Lawrence

\section{References}

1. Lanigan GW. Peptococcus heliotrinreducans, sp. nov., a cytochrome-producing anaerobe which metabolizes pyrrolizidine alkaloids. J Gen Microbiol 1976; 94:1-10. PubMed

2. Associate Editor Validation List no. 10. Validation of the publication of new names and new combinations previously effectively published outside the IJSB. Int J Syst Bacteriol 1983; 33: 438-440.

3. Associate Editor Validation List no. 11. Validation of the publication of new names and new combinations previously effectively published outside the IJSB. Int J Syst Bacteriol 1983; 33: 672-674.

4. Ezaki T, Yabuuchi E. Transfer of Peptococcus heliotrinreducens corrig. to the genus Peptostreptococcus: Peptostreptococcus heliotrinireducens Lanigan 1983 comb. nov. Int J Syst Bacteriol 1986; 36:107-108.

5. Wade WG, Downes J, Dymock D, Hiom SJ, Weightman AJ, Dewhirst FE, Paster BJ, Tzellas N, Coleman B. The family Coriobacteriaceae: reclassification of Eubacterium exiguum (Poco et al. 1996) and Peptostreptococcus heliotrinreducens (Lanigan 1976) as Slackia exigua gen. nov., comb. nov. and Slackia heliotrinireducens gen. nov., comb. nov., and Eubacterium lentum (Prevot 1938) as Eggerthella lenta gen. nov., comb. nov. Int J Syst Bacteriol 1999; 49:595-600. PubMed

6. Murdoch DA. Gram-positive anaerobic cocci. Clin Microbiol Rev 1998; 11:81-120. PubMed

7. Murdoch DA, Mitchelmore IJ. Isolation of Peptostreptococcus heliotinreducens from human polymicrobial abscesses. Lett Appl Microbiol 1989; 9:223-225. doi:10.1111/j.1472765X.1989.tb00331.x

8. Goodacre R, Hiom SJ, Cheeseman SL, Murdoch DA, Weightman AJ, Wade WG. Identification and
Berkeley National Laboratory under contract No. DEAC02-05CH11231, Lawrence Livermore National Laboratory under Contract No. DE-AC52-07NA27344, and Los Alamos National Laboratory under contract No. DEAC02-06NA25396, as well as German Research Foundation (DFG) INST 599/1-1.

discrimination of oral asaccharolytic Eubacterium species by pyrolysis mass spectrometry and artificial neural networks. Curr Microbiol 1996; 32:7784. PubMed doi:10.1007/s002849900014

9. Lee C, Grasso C, Sharlow MF. Multiple sequence alignment using partial order graphs. Bioinformatics 2002; 18:452-464. PubMed doi:10.1093/bioinformatics/18.3.452

10. Castresana J. Selection of conserved blocks from multiple alignments for their use in phylogenetic analysis. Mol Biol Evol 2000; 17:540-552. PubMed

11. Stamatakis A, Hoover P, Rougemont J. A rapid bootstrap algorithm for the RAxML web-servers. Syst Biol 2008; 57:758-771. PubMed doi:10.1080/10635150802429642

12. Liolios K, Mavrommatis K, Tavernarakis N, Kyrpides NC. The Genomes OnLine Database (GOLD) in 2007: status of genomic and metagenomic projects and their associated metadata. Nucleic Acids Res 2008; 36:D475-D479. PubMed doi:10.1093/nar/gkm884

13. Mavrommatis K, Pukall R, Rohde C, Chen F, Sims D, Brettin T, Kuske C, Detter JC, Han C, Lapidus A, et al. Complete genome of Cryptobacterium curtum type strain (12-3T). Stand Genomic Sci 2009; 1: 93-100 doi:10.4056/sigs.12260

14. Saunders E, Pukall R, Abt B, Lapidus A, Galvina Del Rio T, Copeland A, Tice H, Cheng JF, Lucas $\mathrm{S}$, Chen $\mathrm{F}$, et al. Complete genome sequence of Eggerthella lenta type strain (VPI $0255^{\mathrm{T}}$ ). Stand Genomic Sci 2009; 1: 174-182. doi:10.4056/sigs.33592

15. Field D, Garrity G, Gray T, Morrison N, Selengut J, Sterk P, Tatusova T, Thomson N, Allen MJ, Angiuoli SV, et al. The minimum information about 
a genome sequence (MIGS) specification. Nat

Biotechnol 2008; 26:541-547. PubMed

doi:10.1038/nbt1360

16. Woese CR, Kandler O, Wheelis ML. Towards a natural system of organisms: proposal for the domains Archaea, Bacteria, and Eucarya. Proc Natl Acad Sci USA 1990; 87:4576-4579. PubMed $\underline{\text { PubMed doi:10.1073/pnas.87.12.4576 }}$

17. Garrity GM, Holt J. Taxonomic Outline of the Archaea and Bacteria. Bergey's Manual of Systematic Bacteriology, $2^{\text {nd }} \mathrm{Ed}$. In: G.Garrity GM, Boone DR, Castenholz RW Eds. Vol 1 The Archaea, Deeply Branching and Phototrophic Bacteria. 2001 pp. 155-166 PubMed

18. Stackebrandt E, Rainey FA, Ward-Rainey NL. Proposal for a new hierarchic classification system, Actinobacteria classis nov. Int / Syst Bacteriol 1997; 47:479-491.

19. Holdemann Moore LV, Johson JL, Moore WEC. The genus Peptostreptococcus. In: Bergey's Manual of Systematic Bacteriology (ed. PHA Sneath) Vol. 2, 1083-1092.

20. Anonymous. Biological Agents: Technical rules for biological agents, TRBA $466<w w w . b a u a . d e>$.

21. Ashburner M, Ball CA, Blake JA, Botstein D, Butler H, Cherry JM, Davis AP, Dolinski K, Dwight SS, Eppig JT, et al. Gene ontology: tool for the unification of biology. The Gene Ontology Consortium. Nat Genet 2000; 25:25-29. PubMed doi:10.1038/75556

22. List of growth media used at DSMZ: http://www.dsmz.de/microorganisms/media_list.php

23. Wu M, Hugenholtz P, Mavrommatis K, Pukall R, Dalin E, Ivanova N, Kunin V, Goodwin L, Wu M, Tindall BJ, et al.. A phylogeny-driven genomic encyclopedia of Bacteria and Archaea. (In press).

24. Sims D, Brettin T, Detter JC, Han C, Lapidus A, Copeland A, Glavina Del Rio T, Nolan M, Chen F, Lucas $\mathrm{S}$, et al. Complete genome of Kytococcus sedentarius type strain $\left(541^{\mathrm{T}}\right)$. SIGS 2009; 1:1220.

25. Besemer J, Lomsadze A, Borodovsky M. GeneMarkS: a self-training method for prediction of gene starts in microbial genomes. Implications for finding sequence motifs in regulatory regions. Nucleic Acids Res 2001; 29:2607-2618. PubMed doi:10.1093/nar/29.12.2607
26. Markowitz VM, Mavrommatis K, Ivanova NN, Chen IMA, Chu K, Kyrpides NC. Expert Review of Functional Annotations for Microbial Genomes. Bioinformatics 2009; 25:2271-2278. PubMed doi:10.1093/bioinformatics/btp393

27. Pati A, Ivanova N, Mikhailova N, Ovchinikova G, Hooper SD, Lykidis A, Kyrpides NC. GenePRIMP: A Gene Prediction Improvement Pipeline for microbial genomes. (Submitted).

28. Lowe TM, Eddy SR. tRNAscan-SE: a program for improved detection of transfer RNA genes in genomic sequence. Nucleic Acids Res 1997;

25:955-964. PubMed doi:10.1093/nar/25.5.955

29. Lagesen K, Hallin P, Rødland EA, Staerfeldt HH, Rognes T, Ussery DW. RNAmmer: consistent and rapid annotation of ribosomal RNA genes. Nucleic Acids Res 2007; 35:3100-3108. PubMed doi:10.1093/nar/gkm160

30. Griffiths-Jones S, Moxon S, Marshall M, Khanna A, Eddy SR, Bateman A. Rfam: annotating noncoding RNAs in complete genomes. Nucleic Acids Res 2005; 33:D121-D124. $\underline{\text { PubMed }}$ doi:10.1093/nar/gki081

31. Markowitz VM, Szeto E, Palaniappan K, Grechkin Y, Chu K, Chen IMA, Dubchak I, Anderson I, Lykidis A, Mavrommatis K, et al. The Integrated Microbial Genomes (IMG) system in 2007: data content and analysis tool extensions. Nucleic Acids Res 2008; 36:D528-D533. PubMed doi:10.1093/nar/gkm846

32. Karp PD, Paley SM, Romero P. The Pathway Tools Software. Bioinformatics 2002; 18:S225S232. PubMed

33. Caspi R, Foerster H, Fulcher CA, Kaipa P, Krummenacker M, Latendresse M, Paley SM, Rhee SY, Shearer A, Tissier C, et al. The MetaCyc Database of metabolic pathways and enzymes and the BioCyc collection of pathway/Genome Databases. Nucleic Acids Res 2008; 36:D623-D631. PubMed doi:10.1093/nar/gkm900

34. Karp PD, Ouzounis CA, Moore-Kochlacs C, Goldovsky L, Kaipa P, Ahren D, Tsoka S, Darzentas $\mathrm{N}$, Kunin V, Lopez-Bigas N. Expansion of the BioCyc collection of pathway/genome databases to 160 genomes. Nucleic Acids Res 2005; 33:6083-6089. $\quad$ PubMed doi:10.1093/nar/gki892 